\title{
El Trabajo Cooperativo como Herramienta Formativa en los Estudiantes Universitarios
}

\section{Cooperative Work as a Training Tool for University Students}

\author{
Daniel Garrote Rojas ${ }^{1 *}$ \\ Sara Jiménez-Fernández ${ }^{2}$ \\ Nazaret Martínez-Heredia ${ }^{1}$ \\ ${ }^{1}$ Universidad de Granada, España \\ ${ }^{2}$ Complejo Hospitalario de Jaén, España
}

\begin{abstract}
El Espacio Europeo de Educación Superior (EEES) propone trabajar con metodologías docentes activas para que los alumnos puedan alcanzar nuevas competencias. Actualmente los jóvenes deben hacer frente a una sociedad cambiante influida por la eliminación de fronteras a nivel formativo y por la incorporación de nuevas herramientas en su etapa formativa. En este estudio hemos analizado a través del trabajo cooperativo el desarrollo de la competencia interpersonal de trabajo en equipo, como una construcción del conocimiento entre iguales con semejantes habilidades. Se utiliza la rúbrica para analizar cómo los estudiantes perciben el desarrollo de la competencia interpersonal a través del trabajo cooperativo. El estudio se llevó a cabo en la Universidad de Castilla-La Mancha sobre una muestra de estudiantes universitarios. Los resultados obtenidos permiten rechazar la hipótesis de investigación sobre diferencias entre las puntuaciones de la autoevaluación y la coevaluación intragrupo. Se encuentran diferencias estadísticamente significativas en tres ítems, pertenecientes a los dos primeros niveles de dominio, participar y colaborar activamente en las actividades de equipo y contribuir en la consolidación y desarrollo del grupo. Como conclusión, destacamos la edad como variable asociada a una mejor participación por parte de los estudiantes en el desarrollo del grupo. Es importante el trabajo en equipo como herramienta metodológica dentro del aula, desarrollando una serie de competencias dentro de la formación y el desempeño laboral.
\end{abstract}

Descriptores: Estudiante; Autoevaluación; Docente; Proceso de aprendizaje; Grupo.

The European Higher Education Area (EHEA) proposes to work with active teaching methodologies so that students can reach new competences. Currently, young people must face a changing society influenced by the elimination of borders at the training level and the incorporation of new tools in their formative stage. We have analyzed through cooperative work the development of interpersonal teamwork competence, as a construction of knowledge among equals with similar skills. The rubric is used to analyze how students perceive the development of interpersonal competence through cooperative work. The study was carried out at the University of CastillaLa Mancha on a sample of university students. The obtained results allow to reject the hypothesis of research on differences between the scores of the self-evaluation and the intra-group coevaluation. Statistically significant differences are found in three items, belonging to the first two levels of proficiency, participating and collaborating actively in team activities and contributing to the consolidation and development of the group. In conclusion, we highlight age as a variable associated with better participation by students in the development of the group. Teamwork is important as a methodological tool in the classroom, developing a series of competences within the training and work performance.

Keywords: Student; Self-evaluation; Teachers; Learning process; Groups.

*Contacto: danielgarrote@ugr.es

ISSN: $1696-4713$

www.rinace.net/reice/

revistas.uam.es/reice
Recibido: $\quad 4$ de enero 2019

$1^{\text {a }}$ Evaluación: 18 de febrero 2019

$2^{\text {a }}$ Evaluación: 21 de marzo 2019

Aceptado: $\quad 1$ de abril 2019 


\section{Introducción}

A partir de la Declaración de Bolonia de 1999, el Espacio Europeo de Educación Superior (EEES) pretende crear un nuevo modelo educativo orientado a mejorar la convergencia y compatibilidad de los diferentes sistemas universitarios europeos facilitando la movilidad (García-Murrias, Sobrado-Fernández y Fernández-Rey, 2016) y el reconocimiento de los Grados para los estudiantes. Actualmente los jóvenes deben hacer frente a una sociedad cambiante influida por la eliminación de fronteras a nivel formativo y por la incorporación de nuevas herramientas en su etapa formativa y posterior desarrollo profesional. Este nuevo modelo favorece el desarrollo de competencias y habilidades de auto-aprendizaje (Romero y Pérez, 2009).

Dentro de las nuevas herramientas, no sólo hay que considerar las nuevas Tecnologías de la Información y la Comunicación (TIC) sino también las competencias que deben desarrollar los estudiantes. Se producen cambios con la entrada del EEES en la universidad relacionados con la formación de los estudiantes: modelos centrados en la forma de trabajar y afrontar las materias, desarrollar competencias profesionales y un modelo de evaluación donde se favorezca el aprendizaje de los alumnos (Álvarez, Inda y Álvarez, 2012). Las catalogaciones diferencian entre competencias específicas, propias de cada área de estudio y competencias genéricas o transversales, relacionadas con la formación personal del estudiante. En esta investigación nos hemos centrado en las competencias interpersonales, como aquellas capacidades individuales relacionadas con las habilidades sociales, y el trabajo en equipo, de acuerdo con el sistema de clasificación de competencias propuesto en el Proyecto Tuning (González y Wagenaar, 2003). Este nuevo prisma ofrece a los docentes un nuevo rol de mediadores entre las materias a las que deben hacer frente los estudiantes y la autonomía de éstos para asimilar los conocimientos (Ibáñez, 2004). Es fundamental construir nuevas perspectivas educativas que trasciendan el concepto de aprendizaje centrándose en las nuevas realidades sociales, debido al cambio acelerado de las tecnologías, redes sociales y los problemas que afectan a nuestra realidad (Tobón et al., 2015).

\section{Revisión de la literatura}

Las competencias interpersonales pueden desarrollarse gracias a la incorporación de metodologías docentes activas (Corchuelo et al., 2016). La técnica del aprendizaje cooperativo permite modificar la disposición individualista y competitiva del alumno, aumentando su motivación, autonomía y responsabilidad a través de una comunicación activa y constructiva (Fraile, 2008). Cabe resaltar las consideraciones introductorias en torno al trabajo colaborativo; la colaboración es clave para desarrollar el talento de los alumnos, ya que se desarrollan una serie de competencias transformándose en una fuente de estimulación y creatividad, siendo esencial para el apoyo mutuo, se trata de un proceso de respeto hacia las habilidades y conocimiento de los demás, promoviéndose la inclusión (Vázquez Antonio et al., 2017). Si entendemos el aprendizaje cooperativo como un proceso de comunicación orientado a la construcción de conocimiento entre iguales con habilidades semejantes (Tirado, Hernando y Aguaded, 2011), para que la comunicación tenga éxito, se debe estimular a los estudiantes a pensar (Bain, 2006).

Benito y Cruz (2005) consideran que en el epicentro del aprendizaje cooperativo se encuentra el trabajo conjunto de los alumnos para la realización de una tarea, movidos 
tanto por su aprendizaje como por el de sus compañeros, desarrollando habilidades metacognitivas y cognitivas (Salmerón et al., 2010). A través de la acción cooperativa, el grupo podrá alcanzar sus objetivos únicamente si todo el grupo lo ha conseguido (Cejudo, López-Delgado y Latorre, 2015; Fraile, 2008).

Las características del trabajo cooperativo, según Fraile (2008) son los grupos reducidos para priorizar el proceso de interacción, siendo necesario explicar a los estudiantes cómo se debe actuar en el grupo para el desarrollo de habilidades de interacción y resolución de conflictos; planificación y control del tiempo; motivar a los estudiantes a lo largo del proceso; grupos heterogéneos y disponer de los recursos materiales necesarios. Los logros del alumnado se centran en la mejora del rendimiento académico, mejora de la motivación, de las relaciones interpersonales, autoestima, conocer diferentes contextos, etc. (López y Acuña, 2018). Se debe considerar la importancia de una adecuada formación de los estudiantes para trabajar en grupo, conocer qué habilidades han de desarrollar y ser capaces de evaluar tanto su trabajo como el de sus compañeros. Por ello, una de las funciones más relevantes de los docentes es la de enseñar a los estudiantes a saber convivir en grupo, para alcanzar el objetivo común y resolver los problemas que surjan (Pompa et al., 1999).

El trabajo en grupo se puede entender como la adquisición de unos objetivos comunes, pero según indica Winter (2000) son necesarias otras destrezas. Los estudiantes deben trabajar siguiendo una metodología, es decir, identificar un objetivo, planificar una solución y a través de los resultados obtenidos, modificar la planificación. Necesitan comunicar respetando los diferentes puntos de vista sobre un problema, las posibles soluciones o las causas de una dificultad y alcanzar una solución conjunta. Además, deben reconocer el esfuerzo de cada miembro del grupo y valorar las aportaciones que realicen. El profesor universitario se convierte en gestor del proceso de enseñanza-aprendizaje y ofrece oportunidades de aprendizaje individuales y grupales, gestiona las interacciones didácticas y la relación con sus alumnos y establece las condiciones óptimas para el buen clima social en el proceso de enseñanza-aprendizaje (Martínez, 2015), así como ofrecerse ayuda entre ellos para fomentar este aprendizaje (Durán y Flores, 2015).

La motivación resulta clave para que los estudiantes satisfagan sus necesidades de conocimiento (Garrote, Garrote y Jiménez-Fernández, 2016). Para que este aprendizaje no pierda en significatividad y funcionalidad, los alumnos deben ser capaces de relacionar el contenido de la asignatura con las competencias que se pretenden desarrollar y entender la utilidad y aplicabilidad que tendrá el conocimiento que están adquiriendo. Existe un potencial en las experiencias donde los contenidos hacen alusión a situaciones reales (Wall y Shankar, 2008). En este sentido, la participación e implicación personal en los grupos de trabajo revierte en una mejora motivacional enfocada a la actuación profesional (Moreno, Quesada y Pineda, 2010). Diferentes investigaciones muestran la influencia que tiene la motivación en el aprendizaje de los estudiantes (Alonso-Tapia, 2005), donde el docente debe ofrecer al alumnado un entorno en el cual ellos sean los protagonistas de su propio aprendizaje académico y obtengan un beneficio en su proceso de socialización. El rendimiento académico del alumno está relacionado con su inteligencia emocional, cualidades que influyen en su desarrollo personal, académico y profesional (Del Rosal, Moreno-Manso y Bermejo, 2018).

Es necesario resaltar que para la mayoría de los educadores los términos colaborativo y cooperativo poseen significados similares, unos utilizan dichos términos de manera 
intercambiable, otros realizan una clara distinción epistemológica entre ellos. Señalando que la diferencia entre estas dos formas se basa en dos dimensiones, la mutualidad o grado de implicación en la comunicación con los alumnos y la igualdad o igualdad de roles. En el trabajo colaborativo ambas formas son altas, sin embargo, en el trabajo cooperativo la igualdad es alta pero la mutualidad depende del grado en que se anime el aprendizaje grupal y de las recompensas por trabajar en grupo (motivación intrínseca o extrínseca) (Vélez y Olivencia, 2019). A través de esta investigación se plantean tres objetivos relacionados con el trabajo en grupo. Conocer el grado de participación y colaboración en las tareas en grupo a través de la confianza, la cordialidad y la orientación por medio de la autoevaluación y la coevaluación intragrupo. Analizar la consolidación y el desarrollo del grupo a través de la comunicación, la distribución equilibrada de las tareas, el clima interno y la cohesión del mismo mediante la autoevaluación y la coevaluación intragrupo y, por último, identificar la interacción de los miembros del grupo y su orientación hacia un elevado rendimiento por medio de la autoevaluación y la coevaluación intragrupo.

Como hipótesis nula de la investigación planteamos que no existirán diferencias en la valoración que hacen los estudiantes del trabajo cooperativo a través de las puntuaciones obtenidas en la autoevaluación y la coevaluación intragrupo.

\section{Método}

\section{Diseño y participantes}

La investigación observacional se llevó a cabo con un grupo de sesenta y seis estudiantes universitarios $(\mathrm{N}=66)$ de primer curso del Grado de Maestro en Educación Infantil. La edad media de los alumnos fue de 20 años. Mayoritariamente del género femenino (95,5\%). Se utilizó la rúbrica como herramienta de evaluación, permitiendo al alumnado conocer los criterios que iban a evaluar mediante la explicación de los mismos. Considerada como una herramienta de evaluación formativa debido a que involucra a los estudiantes en el proceso de evaluación de su propio trabajo (autoevaluación) y en el de sus compañeros dentro de cada grupo (coevaluación intragrupo) (Chica, 2011).

Una vez formados los grupos, con tres o cuatro miembros, se les explica en qué consiste el trabajo cooperativo que llevarán a cabo durante una investigación. Buscando la interacción, la planificación de las tareas, la gestión del tiempo y la resolución de los conflictos que pudiesen surgir. Posteriormente, se les explica la actividad que tendrían que llevar a cabo como investigación, el desarrollo de un estudio de un caso real sobre alumnos con dificultades de aprendizaje. Como indican Andreu-Andrés y García-Casas (2014), la elección del tipo de trabajo, se llevó a cabo por el docente según el perfil de los estudiantes y sus objetivos. La decisión de realizar el trabajo cooperativo frente al trabajo colaborativo, se debe a que los estudiantes pertenecen al primer curso del Grado y el profesor fue el responsable de estructurar el proceso (Oxford, 1997). La organización de los grupos fue realizada de manera espontánea por los estudiantes, siendo ellos los encargados de organizarse en grupos. En algunos casos fue necesaria la intervención del profesor para organizar a los alumnos que no tenían grupo. La asignatura que cursan los alumnos se rige bajo el EEES con el sistema de créditos ECTS, en los cuales, a través de la guía de la asignatura, se planifica tiempo para la formación de los mismos dentro y fuera del aula. En ambas situaciones, los alumnos deben rellenar unas sesiones de trabajo, donde recogen información sobre el tiempo dedicado ese día al trabajo de investigación, la tarea 
pendiente de la sesión anterior, qué tienen organizado hacer y qué han acordado para la siguiente sesión. A lo largo de los tres meses siguientes se realizaron, dentro del aula, dos seminarios presenciales con cada grupo de forma individualizada, con el fin de guiar a los estudiantes en su trabajo, retroalimentarlos para que adquirieran las destrezas necesarias y guiarlos en el trabajo de investigación. Una vez terminado el proceso de investigación, con la entrega del trabajo de estudio de caso, y tras la exposición del mismo al profesor y todos los compañeros de clase, los estudiantes se autoevaluaron, a través de la rúbrica, y llevaron a cabo la coevaluación intragrupo.

\section{Variables e instrumentos}

Para Panadero y Jonsson (2013), la rúbrica está considerada como la metodología más eficaz en la autoevaluación por sus tres características principales: ofrece unos criterios para la evaluación de las metas a través de los objetivos propuestos, utiliza diferentes niveles de ejecución de las tareas mediante las escalas de valoración y permite a los estudiantes conocer qué grado de ejecución han alcanzado con su trabajo durante el proceso de aprendizaje.

Como instrumento de análisis de la competencia del trabajo en grupo para la autoevaluación y la coevaluación intragrupo se ha utilizado la rúbrica del trabajo en equipo propuesta en Alsina y otros (2013). La rúbrica está desglosada en tres niveles de dominio y cada uno de estos niveles en diferentes indicadores. El Primer nivel de dominio, participar y colaborar activamente en las actividades de equipo, fomentando la confianza, la cordialidad y la orientación en el trabajo conjunto. Esta variable está compuesta por cinco indicadores: entrega del trabajo en el plazo fijado (ítem 1), intervención en la definición de los objetivos del trabajo (ítem 2), colaboración en la definición y en la distribución de las tareas del trabajo en grupo (ítem 3), compartir con el equipo el conocimiento y la información (ítem 4) e implicación en los objetivos del grupo y retroalimentación constructiva (ítem 5). El Segundo nivel de dominio, contribuir en la consolidación y desarrollo del grupo, favoreciendo la comunicación, la distribución equitativa de las actividades, un buen ambiente interno y la cohesión; compuesto por cuatro indicadores: aceptación y nombramiento de las normas del grupo (ítem 6), contribución al establecimiento y a la aplicación de los procesos del trabajo en equipo (ítem 7), actuación para afrontar los conflictos y su cohesión (ítem 8) y valoración de la colaboración del trabajo en equipo (ítem 9). El Tercer nivel de dominio, coordinar los grupos de trabajo buscando la interacción de los componentes y su orientación buscando un alto rendimiento. Formado por cuatro indicadores: colaboración en la definición y en la distribución de las tareas del trabajo en equipo (ítem 10), propuesta al grupo de objetivos (ítem 11), actuación para afrontar los conflictos del equipo y su cohesión (ítem 12) y promoción de la implicación en la gestión y funcionamiento del equipo (ítem 13).

En el proceso de evaluación de la rúbrica se han utilizado puntuaciones entre 1 y 4 tanto para la autoevaluación como para la coevaluación intragrupo. Cada indicador tiene cuatro alternativas de respuesta, estos descriptores van desde el incumplimiento del indicador (puntuación 1) hasta su total cumplimiento (puntuación 4).

\section{Procedimiento}

Se realiza un análisis descriptivo mediante el uso de medidas de tendencia central, medidas de dispersión y medidas de posición del valor que alcanza cada ítem respondido por los alumnos. Tras comprobar que estos siguen una distribución normal (Test de 
Kolmogorov-Smirnov), se realizó una comparación de medias independientes (ANOVA) para cada uno de los diferentes ítems que conforman la rúbrica sobre el trabajo en grupo entre la autoevaluación $(\mathrm{N}=66)$ y la coevaluación intragrupo $(\mathrm{N}=182)$, con un nivel de significación establecido de $p<0,05$. También se describe el factor de interacción de edad mediante la nueva comparación de datos por extractos, procediéndose a la comparación de las evaluaciones realizadas (autoevaluación, coevaluación intragrupo y valoración global), según dos rangos de edad, los menores de 21 años (78,8\% de la muestra) y los mayores de 21 años (21,2\% de la muestra). El paquete estadístico utilizado para llevar a cabo el análisis fue el Statistical Package for the Social Science (SPSS) versión 20.0.

\section{Resultados}

Los participantes en la investigación fueron 66 estudiantes de los cuales el 95,5\% son mujeres, con una edad media de 20,24 $\pm 4,49$, el $78,8 \%$ de los participantes se encuentran entre los 18 y los 20 años. En el cuadro 1 se muestran los porcentajes de respuesta en cada uno de los descriptores de cada ítem relacionado con los niveles de dominio, la media y la desviación típica. Se engloba tanto la autoevaluación como la coevaluación intragrupo. La puntuación media obtenida en los trece ítems que forman la rúbrica oscila entre 3,43 y 3,95, haciendo referencia al ítem 11: "Propuesta al grupo de objetivos ambiciosos" y el ítem 1: "Entrega del trabajo en el plazo fijado". La desviación típica ha sido menor $(0,345)$ en el ítem 1 y mayor $(0,661)$ en el ítem 8: "Actuación para afrontar los conflictos y su cohesión”.

Cuadro 1. Resultados estadísticos globales

\begin{tabular}{|c|c|c|c|c|c|c|}
\hline \multirow{2}{*}{ ÍTEMS } & \multicolumn{4}{|c|}{ PORCENTAJE } & \multirow{2}{*}{ MEdia } & \multirow{2}{*}{$\begin{array}{c}\text { DESVIACIÓN } \\
\text { TÍPICA } \\
\end{array}$} \\
\hline & 1 & 2 & 3 & 4 & & \\
\hline Ítem 1 & 0,8 & 1,2 & 0,0 & 98,0 & 3,95 & 0,345 \\
\hline Ítem 2 & 1,2 & 3,2 & 26,6 & 69,0 & 3,63 & 0,609 \\
\hline Ítem 3 & 1,2 & 3,6 & 31,5 & 63,7 & 3,58 & 0,625 \\
\hline Ítem 4 & 1,2 & 1,2 & 36,0 & 61,5 & 3,58 & 0,585 \\
\hline Ítem 5 & 1,2 & 0,8 & 41,3 & 56,7 & 3,53 & 0,582 \\
\hline Ítem 6 & 0,8 & 0,8 & 32,5 & 65,8 & 3,63 & 0,549 \\
\hline Ítem 7 & 0,4 & 0,8 & 34,6 & 64,1 & 3,62 & 0,527 \\
\hline Ítem 8 & 2,1 & 3,0 & 35,9 & 59,1 & 3,52 & 0,661 \\
\hline Ítem 9 & 1,3 & 2,1 & 27,0 & 69,6 & 3,65 & 0,589 \\
\hline Ítem 10 & 1,7 & 0,8 & 41,8 & 55,7 & 3,51 & 0,608 \\
\hline Ítem 11 & 1,3 & 1,3 & 50,2 & 47,3 & 3,43 & 0,590 \\
\hline Ítem 12 & 0,4 & 2,5 & 35,4 & 61,6 & 3,58 & 0,566 \\
\hline Ítem 13 & 0,8 & 2,5 & 36,7 & 59,9 & 3,56 & 0,591 \\
\hline
\end{tabular}

Fuente: Elaboración propia.

El porcentaje de sujetos que han obtenido una puntuación mayor en la evaluación (puntuación 4) se da, de forma descendente, en los ítem 1: "Entrega del trabajo en el plazo fijado", ítem 9: "Valoración de la colaboración del trabajo en equipo", ítem 2: "Intervención en la definición de los objetivos del trabajo", ítem 6: "Aceptación y nombramiento de las normas del grupo" y el ítem 7: "Contribución al establecimiento y a la aplicación de los procesos del trabajo en equipo", oscilando entre el $98 \%$ y $64,1 \%$. 
Respecto a los ítems más y menos valorados, se han llevado a cabo tres diferenciaciones en la descripción de las puntuaciones, en primer lugar, la puntuación global, que recoge las puntuaciones obtenidas de la autoevaluación y la coevaluación intragrupo. En segundo lugar, la puntuación obtenida por la autoevaluación y finalmente, la coevaluación intragrupo.

Los ítems más valorados, en la puntuación global, de forma decreciente son, en primer lugar, el ítem 1: "Entrega del trabajo en el plazo fijado" con 980 puntos. El ítem 2: "Intervención en la definición de los objetivos del trabajo" con 901 puntos. El ítem 3: "Colaboración en la definición y en la distribución de las tereas del trabajo en grupo" con 887 puntos. Los ítems más valorados, relacionados con las puntuaciones de la autoevaluación, de forma decreciente son el ítem 1: "Entrega del trabajo en el plazo fijado" con 264 puntos. El ítem 3: "Colaboración en la definición y en la distribución de las tereas del trabajo en grupo" con 247 puntos. El ítem 2: "Intervención en la definición de los objetivos del trabajo" con 246 puntos. Los ítems más valorados, relacionados con las puntuaciones de los compañeros, de forma decreciente son el ítem 1: "Entrega del trabajo en el plazo fijado" con 716 puntos. El ítem 2: "Intervención en la definición de los objetivos del trabajo" con 655 puntos. El ítem 4: "Compartir con el equipo el conocimiento y la información" con 641 puntos.

Los ítems con una puntuación menor, en la puntuación global, de forma creciente son, en primer lugar, el ítem 11: "Propuesta al grupo de objetivos ambiciosos" con 814 puntos. El ítem 10: "Colaboración en la definición y en la distribución de las tareas del trabajo en equipo" con 833 puntos. El ítem 8: "Actuación para afrontar los conflictos del equipo y su cohesión” con 834 puntos. Los ítems menos valorados, relacionados con las puntuaciones de la autoevaluación, de forma creciente son el ítem 11: "Propuesta al grupo de objetivos ambiciosos" con 220 puntos. El ítem 10: "Colaboración en la definición y en la distribución de las tareas del trabajo en equipo" con 225 puntos". El ítem 13: "Promoción de la implicación en la gestión y funcionamiento del equipo" con 227 puntos. Los ítems menos valorados, relacionados con las puntuaciones de los compañeros, de forma creciente son el ítem 11: "Propuesta al grupo de objetivos ambiciosos" con 594 puntos. El ítem 8: "Actuación para afrontar los conflictos del equipo y su cohesión” con 606 puntos. El ítem 10: "Colaboración en la definición y en la distribución de las tareas del trabajo en equipo" con 608 puntos.

En el cuadro 2 se realiza la comparación de las medias obtenidas teniendo en cuenta quién ha realizado la valoración de cada estudiante. Por un lado, está la autoevaluación, que es la valoración que cada estudiante hace de sí mismo a lo largo de los trece ítems, y la coevaluación intragrupo, valoración de cada estudiante sobre los miembros de su grupo.

Se obtienen diferencias estadísticamente significativas entre las valoraciones obtenidas entre la autoevaluación y la coevaluación intragrupo en tres ítems, el ítem 3: "Colaboración en la definición y en la distribución de las tereas del trabajo en grupo" (3,74 $\pm 0,47 ; 3,52 \pm 0,66 ; p=0,012)$, el ítem 7: "Contribuir al establecimiento y a la aplicación de los procesos del trabajo en equipo" (3,75 $\pm 0,44 ; 3,58 \pm 0,56 ; p=0,032)$ y el ítem 9: "Valoración de la colaboración del trabajo en equipo" (3,81 $\pm 0,43 ; 3,59 \pm 0,63 ; p=0,012)$.

El ítem 3 pertenece al primer nivel, que busca la participación y colaboración activa en las tareas del equipo y fomentar la confianza, la cordialidad y la orientación en el trabajo que llevan a cabo. Por tanto, la opinión que tiene el estudiante difiere de la de sus compañeros 
de grupo en relación a la colaboración que hay en la definición y distribución de las tareas de sí mismo frente a la de sus compañeros. Los ítems 7 y 9, al segundo nivel, que hace referencia a la contribución en la consolidación y desarrollo del equipo, buscando la comunicación, una distribución equilibrada de la tarea y un buen clima interno y la cohesión del mismo. Apareciendo diferencias entre la propia opinión y la de sus compañeros en relación a la contribución que se hace en el proceso de trabajo en equipo.

Cuadro 2. Evaluación de la participación en el grupo según sea ésta, Autoevaluación y Coevaluación intragrupo

\begin{tabular}{lccc}
\hline \multicolumn{1}{c}{ Ítems } & $\begin{array}{c}\text { AUTOEVALUACIÓN } \\
(\mathbf{N}=\mathbf{6 6})\end{array}$ & $\begin{array}{c}\text { COEVALUACIÓN INTRAGRUPO } \\
(\mathbf{N}=\mathbf{1 8 2})\end{array}$ & VALOR DE $\boldsymbol{p}$ \\
\hline Ítem 1 & $4,00 \pm 0,00$ & $3,93 \pm 0,40$ & 0,184 \\
Ítem 2 & $3,73 \pm 0,45$ & $3,60 \pm 0,66$ & 0,143 \\
Ítem 3 & $3,74 \pm 0,47$ & $3,52 \pm 0,66$ & 0,012 \\
Ítem 4 & $3,68 \pm 0,47$ & $3,54 \pm 0,62$ & 0,095 \\
Ítem 5 & $3,62 \pm 0,49$ & $3,50 \pm 0,61$ & 0,158 \\
Ítem 6 & $3,70 \pm 0,46$ & $3,61 \pm 0,58$ & 0,270 \\
Ítem 7 & $3,75 \pm 0,44$ & $3,58 \pm 0,56$ & 0,032 \\
Ítem 8 & $3,62 \pm 0,58$ & $3,48 \pm 0,69$ & 0,161 \\
Ítem 9 & $3,81 \pm 0,43$ & $3,59 \pm 0,63$ & 0,012 \\
Ítem 10 & $3,57 \pm 0,50$ & $3,49 \pm 0,64$ & 0,389 \\
Ítem 11 & $3,49 \pm 0,50$ & $3,41 \pm \pm 0,62$ & 0,368 \\
Ítem 12 & $3,68 \pm 0,47$ & $3,55 \pm 0,59$ & 0,101 \\
Ítem 13 & $3,60 \pm 0,52$ & $3,54 \pm 0,61$ & 0,470 \\
\hline
\end{tabular}

Fuente: Elaboración propia.

Igualmente sucede con la valoración que se hace sobre la colaboración del trabajo en equipo, en relación a la propia percepción y a la que hacen sus compañeros de grupo sobre ellos. En el tercer nivel, que hace referencia a la dirección de grupos de trabajo, donde se busca la interacción de sus miembros y su orientación hacia un alto rendimiento, no aparecen diferencias significativas entre sus ítems en cuanto a la valoración que hace el sujeto de sí mismo frente a cómo le valoran sus compañeros de grupo.

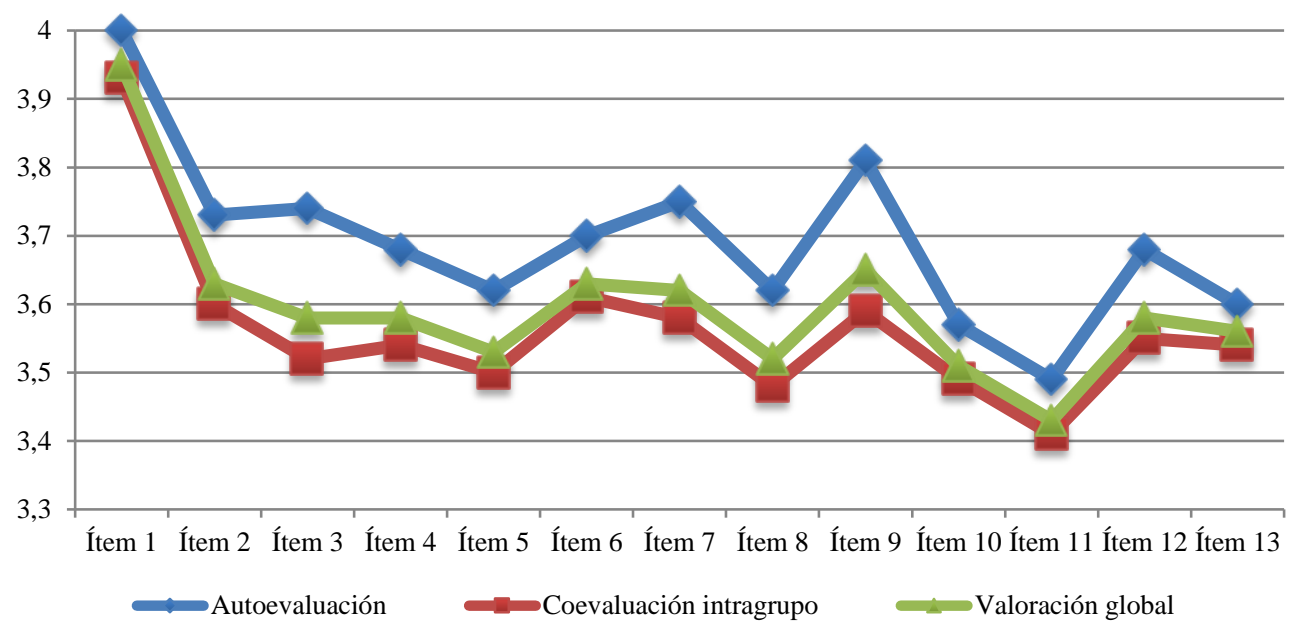

Figura 1. Evaluaciones

Fuente: Elaboración propia. 
En la figura 1 se muestra las puntuaciones que han obtenido los estudiantes en las valoraciones que han llevado a cabo en cada ítem, diferenciando entre autoevaluación, coevaluación intragrupo y evaluación global.

En todos los ítems, la puntuación de la evaluación que los estudiantes hacen de su participación en el grupo es mayor que la puntuación de la evaluación que hacen sus compañeros dentro de cada grupo. Hay una discrepancia entre la valoración que hacen los estudiantes sobre su propio trabajo o contribución al trabajo y la que hacen sus compañeros en todos los ítems, siendo mayor en los ítems 3,7 y 9, donde hemos comentado anteriormente que aparecen diferencias estadísticamente significativas.

Considerando que el $78,8 \%$ de la muestra es menor de 21 años, con una edad media de 18,67 años y que el $21,2 \%$ de la muestra es mayor de 21 años con una edad media de 25,77 años, buscamos conocer si hay discrepancias en las diferentes modalidades de evaluación (autoevaluación, coevaluación intragrupo y valoración global). En la figura 2, en la comparación de datos por extractos de edad, en la autoevaluación, el grupo de edad mayor de 21 años es superior en nueve de los trece ítems. Observamos que entre el ítem 3 al 10 y el ítem 13 las puntuaciones obtenidas por la muestra menor de 21 años son inferiores a la muestra con una edad mayor de 21 años. En el resto de ítems obtienen similar puntuación, los cuales hacen referencia a la aceptación de las normas y su cumplimiento por parte de todos los sujetos de la investigación, la contribución y aplicación en los procesos del trabajo en equipo, la propuesta al grupo de objetivos ambiciosos y el afrontamiento miento de los conflictos y su cohesión. Por tanto, la edad no es un elemento relevante en estos casos.

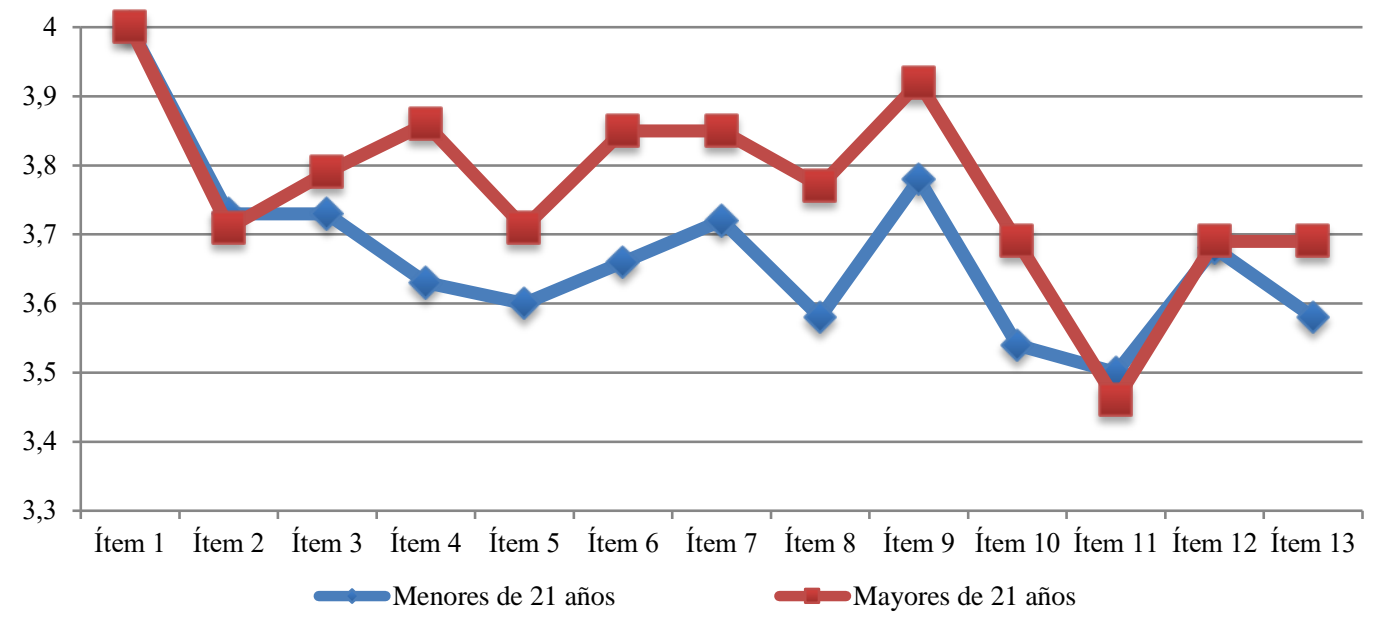

Figura 2. Autoevaluación por extractos de edad Fuente: Elaboración propia.

En la figura 3, las puntuaciones obtenidas por la muestra de edad inferior a 21 años es ligeramente mayor frente a la muestra mayor de 21 años todos los ítems excepto el 6, 7, 8 y 12. Obteniendo puntuaciones similares en los ítems 6, 7 y 12. Por tanto, todos los sujetos, sin diferencia de edad, en la valoración que hacen de cada uno de los miembros del grupo, puntúan de forma similar en los ítems relacionados con la aceptación y cumplimiento de las normas, contribución al establecimiento y aplicación de los procesos del trabajo en equipo y la actuación que tienen para afrontar los conflictos y fomentar la cohesión del grupo. 


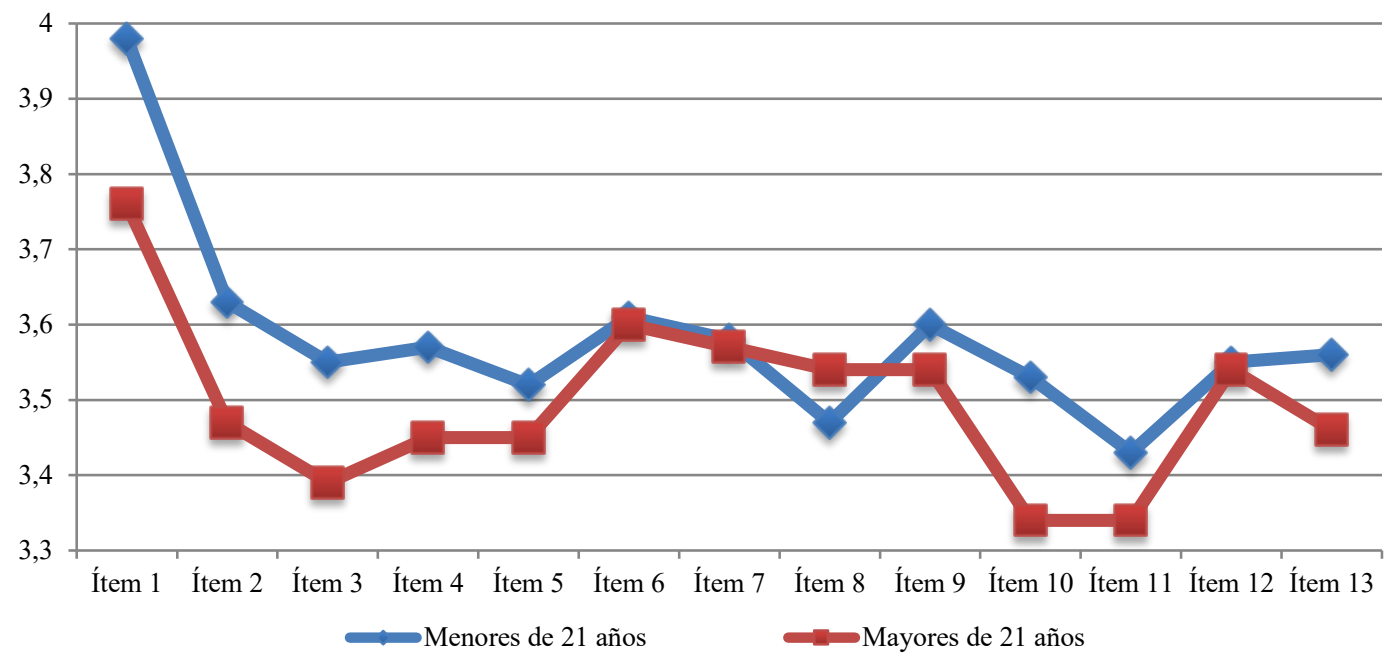

Figura 3. Coevaluación intragrupo por extractos de edad

Fuente: Elaboración propia

En la figura 4, la valoración global, incluye tanto las puntuaciones de la autoevaluación como de la coevaluación intragrupo, diferenciando la muestra por edades. Siendo las puntuaciones mayores en los ítems 1, 2, 3, 10, 11 y 13 en la muestra menor de 21 años, relacionados con el primer nivel (la participación y colaboración activa en las tareas del equipo y el fomento de la confianza, la cordialidad y la orientación en el trabajo conjunto) y tercer nivel (dirección de grupos de trabajo, asegurando la interacción de los miembros y su orientación hacia un elevado rendimiento). Una puntuación similar en los ítems 4, 5 , 9 y 12. Mientras que la muestra mayor de 21 años obtiene mayor puntuación en los ítems 6, 7 y 8, relacionados con el segundo nivel (la contribución a la consolidación y al desarrollo del equipo, favoreciendo la comunicación, la distribución equilibrada de las tareas, el clima interno y la cohesión).

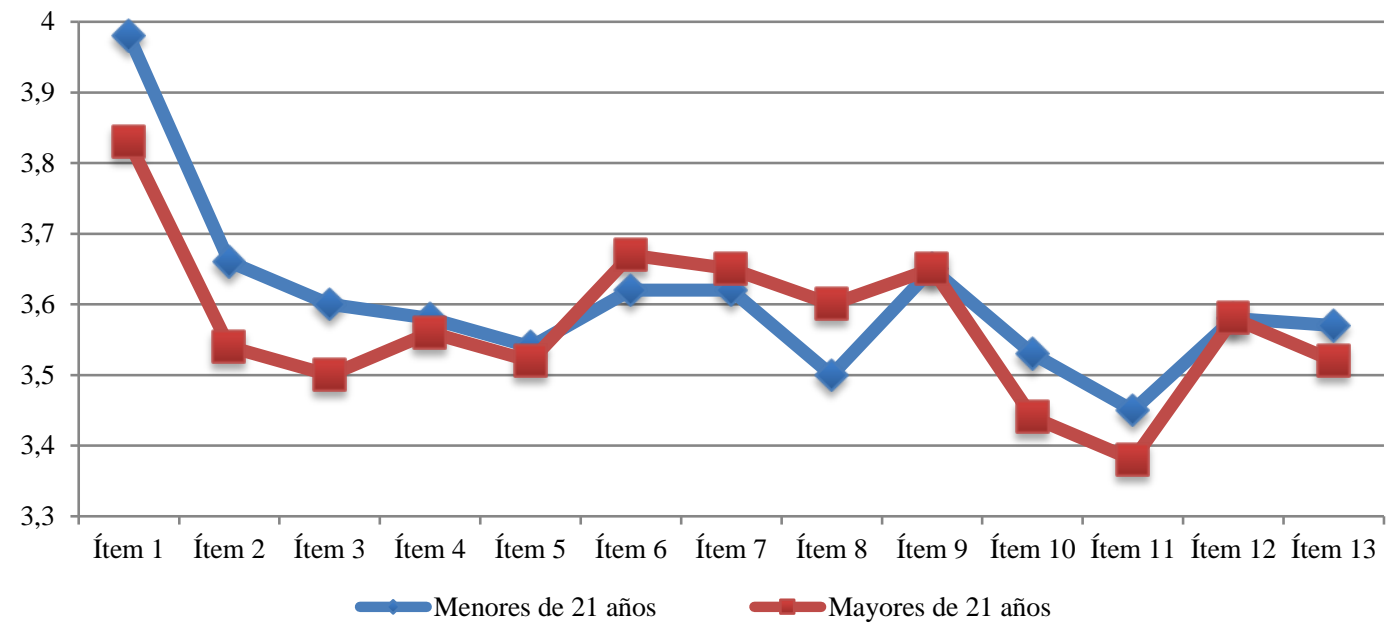

Figura 4. Valoración global por extractos de edad Fuente: Elaboración propia. 


\section{Discusión y conclusiones}

Los resultados que hemos obtenido permiten refutar la hipótesis nula al no existir diferencias en la valoración de los estudiantes del trabajo cooperativo a través de las puntuaciones entre autoevaluación y coevaluación intragrupo. Obteniéndose mayores puntuaciones en la autoevaluación de los estudiantes. De los trece ítems analizados, se obtienen diferencias estadísticamente significativas en tres ítems, el ítem 3: "Colaboración en la definición y en la distribución de las tareas del trabajo en grupo", ítem 7: "Contribución al establecimiento y a la aplicación de los procesos del trabajo en equipo" y el ítem 9: "Valoración de la colaboración del trabajo en equipo". Una vez establecidos los objetivos del grupo, se discute la planificación y distribución de tareas y cómo realizar el trabajo. El ítem 3 valora si se lleva a cabo una actuación activa en este apartado por parte de los integrantes del grupo. El ítem 7 está relacionado con el comportamiento del estudiante dentro del grupo, enfatiza sobre su contribución en la definición de los pasos que debe llevar a cabo el grupo. Por último, el ítem 9 se centra en analizar el comportamiento durante la interacción del estudiante con el resto del grupo, si muestra una actitud proactiva en la valoración de su trabajo.

Los resultados obtenidos en las evaluaciones de autoevaluación y coevaluación intragrupo pueden relacionarse con la Teoría de la Atribución. La satisfacción de los estudiantes por haber realizado el trabajo se vincula más a la intervención individual que grupal. Coincidiendo con Pérez y Poveda (2010), cuando el estudiante atribuye el rendimiento en sus resultados al grado de implicación.

Para la autoevaluación estratificada por edad, los sujetos mayores de 21 años muestran una mayor puntuación en los ítems relacionados con la contribución a la consolidación y desarrollo del equipo. Podemos relacionarlo con una mayor capacidad de liderazgo en estos sujetos. En la coevaluación intragrupo y valoración global según extracto de edad, los mayores de 21 años obtienen mayores puntuaciones en el primer nivel de la rúbrica, la participación y colaboración en las tareas del equipo fomentando un buen ambiente. La edad de los sujetos parece ser un elemento influyente positivamente en el desarrollo del grupo, a mayor edad, mayor participación, colaboración y contribución al grupo.

El uso de la autoevaluación favorece la implicación, motivación y reflexión sobre su propio aprendizaje. Les ayuda a entender mejor el rol que desempeña el profesorado e interactuar con él. Mientras que con la coevaluación intragrupo se fomenta la interactuación con los compañeros del propio grupo (Carless, Joughin y Liu, 2006).

Con respecto a la formación por competencias, la capacidad de enjuiciar sobre el propio avance del alumno, a través de la autoevaluación, es parte esencial en todas las competencias (San Martín, Jiménez y Jerónimo, 2016). Diferentes autores (De Miguel, 2005; Fernández, 2010) ensalzan que en el proceso de enseñanza-aprendizaje el alumno debe auto-administrarse en la evaluación. Las actividades de evaluación proporcionan un beneficio al estudiante en el desarrollo de su conocimiento y en una mayor disposición para futuros aprendizajes fuera del contexto universitario (Boud y Falchikov, 2006).

Para los estudiantes, realizar el trabajo de investigación marcado ha tenido unas consecuencias en su formación y en la gestión de la asignatura por parte del profesor:

- Favorece la comunicación entre profesor-alumno y entre los alumnos. 
- El uso de diferentes recursos TIC facilitan el intercambio de información para aquellos alumnos que no pueden asistir a algún seminario con el profesor o sesión de trabajo con los compañeros, permitiéndoles disponer de la información actualizada.

- Mejora en la organización del trabajo de investigación, porque la estructura del mismo estaba clara para todos los miembros, por las indicaciones del profesor, y eso propicia una mejora en la planificación y gestión del tiempo.

- Favorece la motivación de los estudiantes al ver cumplida cada una de las partes del trabajo, culminando finalmente con la exposición del mismo.

- Además de trabajar la competencia de trabajo en equipo, donde los estudiantes han participado y colaborado de forma activa en la realización del estudio de caso, también se ha desarrollado la competencia "aprender a aprender", ya que los estudiantes han tenido que gestionar el conocimiento que tenían sobre el tema, organizar la búsqueda y el desarrollo de conceptos.

A partir de los objetivos planteados en la investigación y los resultados obtenidos sobre los tres niveles de dominio, hemos podido conocer, en primer lugar, el grado de participación y colaboración en las tareas en grupo a través de la confianza, la cordialidad y la orientación en el trabajo en grupo por medio de la autoevaluación y la coevaluación intragrupo. Hemos podido analizar el alto grado de satisfacción de entrega del trabajo en el plazo fijado, así como el alto grado de cumplimiento en la elaboración de las tareas asignadas. Se produce una activa intervención y participación de los componentes de los grupos en la elaboración de los objetivos de trabajo, permitiendo identificar cómo los estudiantes actúan proactivamente en el planteamiento, discusión y definición de los objetivos del trabajo que se ha responsabilizado el grupo. La alta participación por parte de los miembros de cada grupo en la planificación de las tareas fomenta la organización y distribución de las mismas. Se comparte con el grupo los conocimientos y la información, asumiéndose así los objetivos del grupo. Esta situación fomenta un diálogo constructivo donde se aceptan las opiniones de los demás.

En segundo lugar, analizamos la consolidación y el desarrollo del grupo mediante su elevada aceptación y cumplimiento de las normas para mejorar el funcionamiento del mismo mediante la autoevaluación y la coevaluación intragrupo. Después de definir los objetivos y cómo van a ser alcanzados, resulta importante llevar a cabo un seguimiento sobre qué actitud tiene cada uno de los integrantes del grupo en la aceptación de los objetivos colectivos y del cumplimiento de las tareas asignadas. De esta forma se aplican y mejoran los procesos del grupo mediante la distribución equitativa de las tareas, donde se favorece la actuación positiva en el clima interno, mediante la resolución de conflictos para evitarlos y solucionarlos si se producen. Coordinar y convivir dentro del grupo es esencial, independientemente de los objetivos y tareas de trabajo, dando importancia al trabajo en grupo y favoreciendo así la cohesión del mismo.

Por último, identificamos la interacción que llevan a cabo los miembros del grupo y su orientación hacia un elevado rendimiento mediante una alta planificación en los plazos, distribuyendo las tareas según las habilidades de cada miembro por medio de la autoevaluación y la coevaluación intragrupo. Se propusieron objetivos claros al grupo con visión de futuro. Se analizó así la intención de cada miembro del grupo para ir más allá de los retos que se han planteado en él; la actuación en la resolución de conflictos ha permitido 
evitarlos y solucionarlos; y la gestión adecuada sobre el funcionamiento del grupo, buscando el compromiso personal de todos sus miembros. Además de valorar los conocimientos y las actividades que plantea el grupo, hemos conocido el comportamiento de cada miembro en la gestión y funcionamiento del mismo y cómo es su implicación.

A través de la utilización del trabajo en grupo como herramienta metodológica conseguimos ejercer una competencia que deben adquirir los estudiantes no sólo para su etapa formativa, sino para un correcto desempeño laboral, como señalan Ibarra, Rodríguez y Gómez (2012). Se logra que trabajen, desarrollen y fortalezcan otras competencias como son saber comunicar sus ideas, analizar una propuesta ajena, alcanzar acuerdos y resolver conflictos que puedan surgir. Se busca desarrollar una actitud en ellos, durante la formación universitaria, donde alcancen la integración en la sociedad, pero no solamente en relación a las demandas sociales sino en cuanto a valores humanos (Aramendi et al., 2014). Se fomenta una actitud crítica-constructiva a lo largo del proceso enseñanzaaprendizaje (Iranzo, Gimeno y Sánchez, 2012), donde el profesorado asume mayoritariamente el trabajo, siendo el referente en el proceso de enseñanza-aprendizaje, pero aparece la distribución en responsabilidad entre profesor y alumno (HernándezSellés, González-Sanmamedy y Muñoz-Carril, 2015).

La mejora en el proceso de enseñanza-aprendizaje a través de un aprendizaje autónomo, profundo y eficaz y tras considerar diferentes investigaciones (Efklides, 2012; Hodgson y Pang, 2012; Panadero, Alonso-Tapia y Huertas, 2012; Sáiz et al., 2012) parece claro que la autoevaluación de los estudiantes facilita el desarrollo de aprendizajes más efectivos considerando la existencia o no de diferencias en la percepción de los estudiantes sobre su adquisición del aprendizaje (Sáiz y Bol, 2014). La retroalimentación que ofrece el docente al estudiante resulta una herramienta con la que modular este proceso. Boud y Falchikov (2006) consideran que a través de la evaluación de las actividades se favorece al conocimiento y una mejor predisposición en el aprendizaje, llamándolo, evaluación sostenible. Se hace necesario, no solamente la evaluación final del trabajo, sino el seguimiento del mismo a través de seminarios que faciliten la comunicación entre profesor y alumno y exista un marco donde los alumnos puedan intercambiar opiniones.

\section{Limitaciones y fortalezas}

Las dificultades a lo largo del trabajo hacen referencia al proceso de evaluación por parte del estudiante en el que tiene que valorar su participación y la de sus compañeros como son la falta de objetividad por juicio de valor de un miembro del grupo, por una opinión no compartida o dificultades en la convivencia y el dominio que pueda ejercer un miembro o varios para manipular las decisiones. Dentro del grupo puede haber una difusión en la responsabilidad sobre las fases del proceso de enseñanza-aprendizaje, así como la polarización en las decisiones. Dando lugar a una alteración en las puntuaciones de la coevaluación intragrupo. Con el fin de minimizar el efecto que pudiese tener el sesgo de medición de cada uno de los sujetos en la coevaluación se llevó a cabo la rúbrica de forma anónima, asignando un número aleatorio a cada sujeto y a cada miembro del grupo. Así mismo, al realizarse la formación de algunos grupos de manera espontánea pudo dar lugar a un sesgo de selección, ya que su formación pudo deberse a afinidades entre ellos, frente a otros grupos que fueron organizador por el profesor.

Como fortaleza, destacamos que no se producen perdidas de muestra, todos los estudiantes completaron la rúbrica sobre autoevaluación y coevaluación intragrupo correctamente. 
Así como una valoración muy positiva de la experiencia sobre el trabajo colaborativo realizada como puede observarse en las puntuaciones medias obtenidas.

\section{Futuras líneas}

Como se ha descrito, los dos agentes principales son el docente y el discente. A través del trabajo cooperativo por parte de los estudiantes se puede introducir una nueva vía de aprendizaje, donde el docente tiene una función marcada por el EEES. El aprendizaje cooperativo es una metodología que favorece el desarrollo de competencias necesarias durante el proceso de formación y para el desarrollo laboral, como la comunicación activa, la responsabilidad o la autonomía. Resulta interesante seguir analizando qué elementos pueden favorecer a nivel individual y grupal el desarrollo de estas competencias. Pero también atendiendo a la edad de los sujetos y la influencia que sobre ellos tendría recibir una correcta formación en sus diferentes etapas formativas en compromiso, participación y rendimiento en las relaciones grupales. A través del aprendizaje cooperativo se desarrolla tanto el aprendizaje como la socialización (Gillies, 2014) y provoca en los alumnos un aumento en la confianza en ellos mismos (Pérez y Poveda, 2010). A nivel docente, buscar la implementación de nuevas modalidades de evaluación de los aprendizajes disponibles con el EEES, la autoevaluación y la coevaluación, en una sociedad donde las TIC cada vez tienen más cabida en los jóvenes universitarios (Garrote, JiménezFernández y Serna, 2018). Coincidiendo con Álvarez (2008), el profesor no debe renunciar a su responsabilidad sobre la evaluación, siendo beneficioso compartir dicha tarea con los estudiantes ya que repercute sobre el compromiso y la motivación a lo largo del proceso de enseñanza-aprendizaje. Cuando el estudiante considera que su formación académica se ve influenciada por el nivel de participación que tiene en la evaluación hace que mejore su rendimiento (Cardona, Vélez y Tobón, 2016).

\section{Referencias}

Alonso-Tapia, J. (2005). Motivar en la escuela, motivar en la familia. Madrid: Ediciones Morata.

Alsina, J., Argila, A., Aróztegui, M., Arroyo, F. J., Badia, M., Carreras, A., ... y Vila, B. (2013). Rúbricas para la evaluación de competencias. Barcelona: Ediciones Octaedro.

Álvarez, I. (2008). La coevaluación como alternativa para mejorar la calidad de aprendizaje de los estudiantes universitarios: valoración de una experiencia. Revista Interuniversitaria de Formación del Profesorado, 63, 127-140.

Álvarez, S., Inda, M. y Álvarez, M. (2012). El trabajo cooperativo y la triple evaluación dinamizan la enseñanza-aprendizaje de una lengua extranjera. Revista de Investigación Educativa, 1O(1), $76-87$.

Andreu-Andrés, M. A. y García-Casas, M. (2014). Evaluación del pensamiento crítico en el trabajo en grupo. Revista de Investigación Educativa, 32(1), 203-222. https://doi.org/10.6018/rie.32.1.157631

Aramendi, P., Bujan, K., Garín, S. y Vega, A. (2014). Estudio de caso y aprendizaje cooperativo en la universidad. Profesorado. Revista de Currículum y Formación de Profesorado, 18(1), 413-429.

Bain, K. (2006). Lo que hacen los mejores profesores universitarios. Valencia: Publicaciones de la Universidad de Valencia.

Benito, A. y Cruz, A. (2005). Nuevas claves para la docencia universitaria. Madrid: Narcea. 
Boud, D. y Falchikov, N. (2006). Aligning assessment with long-term learning. Assessment $\Theta^{\circ}$ Evaluation in Higher Education, 31(4), 399-413. https://doi.org/10.1080/02602930600679050

Cardona, S., Vélez, J. y Tobón, S. (2016). Contribución de la evaluación socioformativa al rendimiento académico en pregrado. Revista Educar, 52(2), 423-447.

Carless, D., Joughin, G., Liu, N. F. (2006). How assessment supports learning: Learning-oriented assessment in action. Hong Kong: Hong Kong University Press. https://doi.org/10.5790/hongkong/9789622098237.001.0001

Cejudo, J., López-Delgado, M. L. y Latorre, J. M. (2015). La formación en educación emocional de los docentes: Una visión de los futuros maestros. Revista Española de Orientación y Psicopedagogía, 26(3), 45-62. https://doi.org/10.5944/reop.vol.26.num.3.2015.16400

Chica, E. (2011). Una propuesta de evaluación para el trabajo en grupo mediante rúbrica. Escuela Abierta, 14, 67-81.

Corchuelo, B., Blanco, M. A., López, M. J. y Corrales, N. (2016). Aprendizaje cooperativo interdisciplinar y rúbricas para la mejora del proceso de enseñanza-aprendizaje. @tic Revista de Innovación Educativa, 16, 10-19.

De Miguel, M. (2005). Modalidades de enseñanza centradas en el desarrollo de competencias. Orientaciones para promover el cambio metodológico en el espacio europeo de educación superior. Oviedo: Ministerio de Educación y Ciencia. Universidad de Oviedo.

Del Rosal, I., Moreno-Manso, J. M. y Bermejo, M. L. (2018). Inteligencia emocional y rendimiento académico en futuros maestros de la Universidad de Extremadura. Profesorado. Revista de Currículum y Formación de Profesorado, 22(1), 257-275.

Durán, D. y Flores, M. (2015). Prácticas de tutoría entre iguales en universidades del estado español y de Iberoamérica. REICE. Revista Iberoamericana sobre Calidad, Eficacia y Cambio en Educación, 13(1), 5-17.

Efklides, A. (2012). Commentary: How readily can findings from basic cognitive psychology research be applied in the classroom? Learning and Instruction, 22, 290-295. https://doi.org/10.1016/j.learninstruc.2012.01.001

Fernández, A. (2010). La evaluación orientada al aprendizaje en un modelo de formación por competencias en la educación universitaria. Revista de Docencia Universitaria, 8(1), 11-34. https://doi.org/10.4995/redu.2010.6216

Fraile, A. (2008). El aprendizaje cooperativo como metodología para el desarrollo de los ECTS: Una experiencia de formación del profesorado de educación física. Revista Fuentes, 8, 22-35.

García-Murrias, R., Sobrado-Fernández, L. M. y Fernández-Rey, E. (2016). Análisis de la información orientadora para la movilidad académica en el programa Erasmus. Revista Española de Orientación y Psicopedagogía, 27(1), 67-82. https://doi.org/10.5944/reop.vol.27.num.1.2016.17008

Garrote, D., Garrote, C. y Jiménez-Fernández, S. (2016). Factores influyentes en motivación y estrategias de aprendizaje en los alumnos de grado. REICE. Revista Iberoamericana sobre Calidad, Eficacia y Cambio en Educación, 14(2), 31-44. https://doi.org/10.15366/reice2016.14.2.002

Garrote, D., Jiménez-Fernández, S. y Serna, R. M. (2018). Gestión del tiempo y uso de las TIC en estudiantes universitarios. Píxel-Bit. Revista de Medios y Educación, 53, 109-121. https://doi.org/10.12795/pixelbit.2018.i53.07 
Gillies, R. (2014). Developments in cooperative learning: Review of research. Anales de Psicología, 30(3), 792-801. https://doi.org/10.6018/analesps.30.3.201191

González, J. y Wagenaar, R. (Eds.). (2003). Tuning educational structures in Europe. Informe final. Bilbao: Universidad de Deusto y Universidad de Groningen.

Hernández-Sellés, N., González-Sanmamedy, M. y Muñoz-Carril, P. B. (2015). El rol docente en las ecologías de aprendizaje: Análisis de una experiencia de aprendizaje colaborativo en entornos virtuales. Profesorado. Revista de Currículum y Formación de Profesorado, 19(2), 147163.

Hodgson, P. y Pang, M. (2012). Effective formative e-assessment of student learning: A study on a statistics course. Assessment \& Evaluation in Higher Education, 37(2), 215-225. https://doi.org/10.1080/02602938.2010.523818

Ibáñez, J. S. (2004). Cambios metodológicos con las TIC: Estrategias didácticas y entornos virtuales de enseñanza-aprendizaje. Bordón. Revista de Pedagogía, 56(3), 469-481.

Ibarra, M. S., Rodríguez G. y Gómez M. A. (2012). La evaluación entre iguales: Beneficios y estrategias para su práctica en la universidad. Revista de Educación, 359, 206-231.

Iranzo, E., Gimeno, E. y Sánchez, P. (2012). Estrategias de evaluación de competencias de los alumnos universitarios a través del trabajo grupal. Revista Iberoamericana de Evaluación Educativa, 5(1), 216-229.

López, G. y Acuña, S. (2018). Aprendizaje cooperativo en el aula. Invention, 7(14), 29-38.

Martínez, J. A. (2015). Nuevos roles del profesor y del estudiante universitario en el contexto del espacio europeo de educación superior. Aplicación al área de organización de empresas. Cuadernos de Educación y Desarrollo, 55, 1-34.

Moreno, M. V., Quesada, C. y Pineda, P. (2010). El grupo de trabajo como método innovador de formación del profesorado para potenciar la transferencia del aprendizaje. Revista Española de Pedagogía, 68(246), 281-296.

Oxford, R. (1997). Cooperative learning, collaborative learning and interaction: Three comminicative strands in the lenguaje classroom. Modern Language Journal, 81, 443-456. https://doi.org/10.1111/j.1540-4781.1997.tb05510.x

Panadero, E. y Jonsson, A. (2013). The use of scoring rubrics for formative assessment purposes revisited: A review. Educational Research Review, 9, 129-144. https://doi.org/10.1016/j.edurev.2013.01.002

Panadero, E., Alonso-Tapia, J. y Huertas, J. A. (2012). Rubrics and self-assessment scripts effects on self-regulation, learning and self-efficacy in secondary education. Learning and Individual Differences, 22, 806-813. https://doi.org/10.1016/j.lindif.2012.04.007

Pérez, A. M. y Poveda, P. (2010). Atribuciones causales y aprendizaje cooperativo. Revista Española de Orientación y Psicopedagogía, 21(1), 59-69.

https://doi.org/10.5944/reop.vol.21.num.1.2010.11509

Pompa, A., Mohar, F., Lam, F., Pérez, G., López, E., Falcón, M., ... y Pérez, H. (1999). Contribución al desarrollo del componente investigativo en los estudiantes del primer año de la carrera de medicina veterinaria. Revista Pedagogía Universitaria, 4(3), 36-42.

Romero, M. y Pérez, M. (2009). Como motivas a aprender en la universidad: Una estrategia fundamental contra el fracaso académico en los nuevos modelos educativos. Revista Iberoamericana de Educación, 51, 87-105.

Sáiz, M. C. y Bol, A. (2014). Aprendizaje basado en la evaluación mediante rúbricas en la educación superior. Suma Psicología, 21(1), 28-35. https://doi.org/10.1016/S0121-4381(14)70004-9 
Sáiz, M. C., Montero, E., Bol, A. y Carbonero, M. A. (2012). An analysis of learning competences at the university. Electronic Journal of Research in Educational Psychology, 10(1), 253-270.

Salmerón, H., Gutiérrez-Braojos, C., Rodríguez S. y Salmerón, P. (2010). Influencia del aprendizaje cooperativo en el desarrollo de la competencia para aprender a aprender en la infancia. Revista Española de Orientación y Psicopedagogía, 21(2), 308-319. https://doi.org/10.5944/reop.vol.21.num.2.2010.11534

San Martín, S., Jiménez, N. y Jerónimo, E. (2016). La evaluación del alumnado universitario en el espacio europeo de educación superior. Aula Abierta, 44, 7-14. https://doi.org/10.1016/j.aula.2015.03.003

Tirado, R., Hernando, A. y Aguaded, J. I. (2011). Aprendizaje cooperativo on-line a través de foros en un contexto universitario: Un análisis del discurso y de las redes. Revista Estudios sobre Educación, 20, 49-71.

Tobón, S., González, L., Nambo, J. S., y Vázquez, J. M. (2015). La socioformación: Un estudio conceptual. Paradigma, 36(1), 7-29.

Vázquez Antonio, J. M. V., Mosqueda, J. S. H., Vázquez-Antonio, J., Hernández, L. G. J. y Calderón, C. E. G. (2017). El trabajo colaborativo y la socioformación: Un camino hacia el conocimiento complejo. Educación y Humanismo, 19(33), 334-356. https://doi.org/10.17081/eduhum.19.33.2648

Vélez, A. P. y Olivencia, J. J. (2019). Metodologías cooperativas y colaborativas en la formación del profesorado para la interculturalidad. Tendencias Pedagógicas, 33, 37-46. https://doi.org/10.15366/tp2019.33.003

Wall, S. y Shankar, I. (2008). Adventures in transdisciplinary learning. Studies in Higher Education, 33(5), 551-565. https://doi.org/10.1080/03075070802373008

Winter, R. (2000). Public pedagogy and the responsibility of intellectuals: Youth, littleton, and the loss of innocence. Journal of Composition Theory, 2O(1), 9-42.

\section{Breve CV de los autores}

\section{Daniel Garrote Rojas}

Profesor Contratado Doctor del Departamento de Pedagogía de la Universidad de Granada. Investigador del Grupo de Investigación "Valores Emergentes, Educación Social y Políticas Educativas" (HUM-580). Sus líneas de investigación se centran en el uso de las nuevas tecnologías en los estudiantes y el profesorado, las dificultades de aprendizaje, las estrategias de aprendizaje y la motivación. ORCID ID: https://orcid.org/0000-0002-5970-3646. Email: danielgarrote@ugr.es

\section{Sara Jiménez-Fernández}

Licenciada en Medicina y Facultativa Especialista de Área en Psiquiatría. Doctora por la Universidad de Granada. Venia docendi en la Facultad de Medicina de la Universidad de Granada. Psiquiatra de la Unidad de Salud Mental Infanto-Juvenil del Complejo Hospitalario de Jaén. Investigadora del Grupo de Investigación Psiquiatría y Neurociencias (CTS549). ORCID ID: https://orcid.org/O000-0003-1515-2413. Email: sarajimenezfer@hotmail.com 


\section{Nazaret Martínez-Heredia}

Licenciada en Pedagogía. Máster en Educación Social. Investigación y Desarrollo Profesional. Doctoranda del programa de Ciencias de la Educación de la Universidad de Granada. Profesora e investigadora en formación (FPU) de la Universidad de Granada. Investigadora del Grupo de Investigación "Valores Emergentes, Educación Social y Políticas Educativas" (HUM-580). Interesada en las líneas de investigación de pedagogía crítica, educación social, educación en personas mayores y adultos. ORCID ID: https://orcid.org/0000-0001-7029-7127. Email: nazareth@ugr.es 